\title{
Non-Linear EEG Measures in Meditation
}

\author{
Oldřich Vyšata1,2, Martin Schätz¹, Jakub Kopal1, Jan Burian³, Aleš Procházka1, \\ Kuchyňka Jiří2 ${ }^{2}$ Jakub Hort ${ }^{4,5}$, Martin Vališ ${ }^{2}$ \\ ${ }^{1}$ Department of Computing and Control Engineering, Institute of Chemical Technology, Prague, Czech Republic \\ ${ }^{2}$ Department of Neurology, Faculty of Medicine in Hradec Kralove, Charles University, Hradec Kralove, \\ Czech Republic \\ ${ }^{3}$ Faculty of Informatics and Statistics, University of Economics Prague, Prague, Czech Republic \\ ${ }^{4}$ Memory Disorders Clinic, Department of Neurology, Charles University in Prague, 2nd Faculty of Medicine and \\ University Hospital Motol, Prague, Czech Republic \\ ${ }^{5}$ International Clinical Research Center, St. Anne's University Hospital Brno, Brno, Czech Republic \\ Email: valismar@seznam.cz
}

Received 22 May 2014; revised 7 July 2014; accepted 16 July 2014

Copyright (C) 2014 by authors and Scientific Research Publishing Inc.

This work is licensed under the Creative Commons Attribution International License (CC BY).

http://creativecommons.org/licenses/by/4.0/

(c) (i)

Open Access

\begin{abstract}
In this study, the performance of Sevcik's algorithm that calculates the fractal dimension and permutation entropy as discriminants to detect calming and insight meditation in electroencephalographic (EEG) signals was assessed. The proposed methods were applied to EEG recordings from meditators practicing insight meditation and calming meditation before as well as during both types of meditation. Analysis was conducted using statistical hypothesis testing to determine the validity of the proposed meditation-identifying techniques. For both types of meditation, there was a statistically significant reduction in the permutation entropy. This result can be explained by the increased EEG synchronization, which is repeatedly observed in the course of meditation. In contrast, the fractal dimension (FD) was significantly increased during calming meditation, but during insight meditation, no statistically significant change was detected. Increased FD during meditation can be interpreted as an increase in self-similarity of EEG signals during self-organisation of hierarchical structure oscillators in the brain. Our results indicate that fractal dimension and permutation entropy could be used as parameters to detect both types of meditation. The permutation entropy is advantageous compared with the fractal dimension because it does not require a stationary signal.
\end{abstract}

\section{Keywords}

Permutation Entropy, Fractal Dimension, EEG, Calming Meditation, Insight Meditation

How to cite this paper: Vyšata, O., Schätz, M., Kopal, J., Burian, J., Procházka, A., Jiří, K., Hort, J. and Vališ, M. (2014) Non-Linear EEG Measures in Meditation. J. Biomedical Science and Engineering, 7, 731-738. 


\section{Introduction}

Meditation techniques are often used to influence stress-dependent diseases, such as anxiety, hypertensive disorders or coronary disease, tension headaches or dependencies. However, evidence of successful treatment by meditation in these diseases is weak. One reason may be the difficulty of objective monitoring during meditation and degree of mastery of meditation techniques by individual patients for individual patients.

Longitudinal studies demonstrate that meditation practice significantly changes early stimuli processing, which leads to improved dynamics and flexibility of brain functions related to attention. Improved dynamics and flexibility of brain functions are achieved by a more flexible allocation of attention resources that most likely modulate early processing based on independent stimuli. Three different human brain neuron networks control the functions of wakefulness, orientation and executive control. The right frontal and right parietal cortices and the thalamus are important for maintaining wakefulness. The superior parietal cortex, temporoparietal junction, frontal eye fields and superior colliculus control the orientation function. The anterior cingulate cortex, ventral lateral cortex, prefrontal cortex and basal ganglia are associated with the control of executive processes. If attention is no longer focused on the meditation object, the "default mode" is activated, including the posterior cingulate, medial prefrontal cortex, posterior lateral parietal temporal cortex and parahippocampal gyrus [1]. The cooperation between these brain areas may, to a certain degree, be estimated using the similarities of EEG signals detected by the electrodes placed over these areas. Thanks to its clinical use (see overview in Baer [2] and Didonna [3]), vipassana or insight meditation, especially its secularised version called mindfulness meditation, is an increasingly common subject of research. This study compares the EEG signal complexity measures in experienced meditators (more than 1000 hours of meditation practice) before and during meditation. Under vipassana meditation, both types of attention regulation are used and are complementary to each other. Therefore, this type of meditation is also sometimes called samatha-vipassana. Concentration is necessary for the basic stabilisation of attention. However, in the later stages of meditation practice, a greater emphasis is placed on mindfulness.

Brain activity is spatially coherent, and its degree of freedom is low in the idle state. To activate the various parts of the brain, EEG activity spreads to a large number of neural networks, and the resulting signal can be appropriately modelled by a stochastic system producing linearly correlated noise with a high degree of freedom. Traditionally, EEG signals are considered linear stochastic processes, which have been used for decades as the gold standard in many clinical applications [4]. Some papers [5] show that the degrees of complexity of linearly correlated noise are very similar to measures complexity derived from purely chaotic systems. Measures of fractal dimensions are derived from fractals, which are formally (mathematically) defined. Many real-world phenomena have fractal properties. Therefore, it may be useful to describe them by the fractal dimension. The fractal dimension of the time series cannot be precisely calculated and must be estimated. Various degrees of the fractal dimension are sensitive to the numerical and experimental noise and also to the length of the time series. No physical object is a true fractal, because it cannot have self-similarity at all scales. Therefore, the fractal dimension rather expresses the suitability of describing the properties of fractal modelling. The complexity of the signal can be analyzed directly in the time domain, frequency domain or phase space [6].

The Sevcik method of calculating the fractal dimension has the advantage of small amplitude sensitivity to noise, and the accuracy of estimating the fractal dimension is independent on the length of the evaluated signal [7]. Unlike fractal dimension analysis, using EEG entropy permutation doesn't require stationarity and can be applied to non-stationary EEG signal without segmentation [8]. Measures derived from the theory of non-linear dynamical systems may produce unreliable results for linearly filtered noise [9]. Moreover, the EEG signal is only weakly non-linear [10] [11]. To distinguish the filtered noise from the non-linear signal, surrogate data testing is often used. Unfortunately, this method is reliable only for stationary signals [12], but EEG signals are non-stationary.

Only one study to date has compared concentration and mindfulness within the same group of meditators from the performance spectrum perspective [13]. A similar study of permutation entropy and fractal dimension has not yet been performed. In general, fractal dimension and permutation entropy are considered important parameters and features of EEG signals and biosignals. Studies of EEG complexity during meditation are sporadic. When compared to rest, meditation was accompanied by a focused decrease of dimensional complexity estimates over the midline frontal and central regions [14]. The aim of the present study was to evaluate the non-linearity of EEG signals of two states (before meditation and during meditation). We expected that non-linear analysis would allow objective monitoring during meditation. 


\section{Methods}

\subsection{Participation}

The experiment enrolled meditators practicing insight meditation (samatha-vipassana) as it is practiced in Theravada Buddhism. The prerequisites considered were active meditation experience and the length of meditation experience. Here, meditation experience indicates a formal meditation experience, i.e., in a calm environment, while sitting or walking.

The following meditators were enrolled in the experiment:

1) At the time of the experiment, they had been actively formally practicing at least 2 hours a week (e.g., 30 minutes, 4 days a week).

2) The length of their meditation experience exceeded 1000 hours (e.g., approximately 3 years of one-hour meditations a day, or approximately 4 months of intensive whole-day meditation experience, etc.).

This type of experience allowed us to assume that significant permanent changes (traits) would be apparent in the function and structure of the meditators' brains.

This study included 22 subjects (21 men and 1 woman) with a mean age of 42 (range, 22 - 64) years.

\subsection{Recording Conditions}

EEG data were collected using a 19-channel electrode cap from the following electrode locations: Fp1, Fp2, F3, F4, F7, F8, Fz, C3, C4, T7, T8, Cz, P3, P4, P7, P8, Pz, O1 and O2. The electrodes were referenced to linked earlobes, using a forehead ground. Impedances were maintained below $10 \mathrm{kV}$. The signals were recorded with a digitisation rate of $256 \mathrm{~Hz}$.

\subsection{Procedure}

Each experiment participant gradually underwent five phases. All phases were monitored using EEG. The phases were separated with a sound signal and were as follows:

- Adaptation (idle EEG without meditation), 10 minutes.

- Calming meditation (concentrated attention on breathing, raising and lowering the abdominal wall while breathing in and out), 30 minutes.

- Break, 18 minutes. For the first five minutes of the break, EEG signals were recorded. Then, the participants were allowed to have a slow walk, stretch, etc. During the last three minutes of the break, the participants were repositioned and ready for meditation.

- Insight meditation (open observation of any objects that enter the consciousness moment by moment), 30 minutes.

- Idle EEG with meditation, last 5 minutes.

In total, the experiment lasted 90 minutes.

For detailed instructions please see the Appendix.

\subsection{Data Analysis}

Stored digitised data were zero-phase digitally filtered using a bandpass FIR filter (100 coefficients, Hamming window) of 0.5 - $60 \mathrm{~Hz}$ and a bandstop filter of $49-51 \mathrm{~Hz}$.

\subsection{The Fractal Dimension}

Fractal dimensions can be used to measure the amount of chaos in a time series and thus provide a possible metric for the detection of certain changes from the norm within brain activity. Sevcik's modified method calculates the approximate fractal dimension (D) from a set of $\mathrm{N}$ values $y$ sampled from a waveform between time $t_{\min }$ and $t_{\max }$ with the sampling interval $\delta$. The waveform was subjected to a double linear transformation that maps it into a unit square. The normalised abscissa of the square is $x_{i}^{\prime}$, and the normalised ordinate is $y_{i}^{\prime}$, with both of them defined as

$$
x_{i}^{\prime}=\frac{x_{i}}{x_{\max }}
$$




$$
y_{i}^{\prime}=\frac{y_{i}-y_{\min }}{y_{\max }-y_{\min }}
$$

where $x_{\max }$ is the maximum $x_{i}$, and $y_{\min }$ and $y_{\max }$ are the minimum and maximum $y_{i}$. The fractal dimension of the waveform (Ö) is then approximated by $D$ as

$$
\Phi \approx D=1+\frac{\ln (L)}{\ln \left(2 . N^{\prime}\right)}
$$

where $L$ is the length of the curve in the unit square and $N^{\prime}=N-1$ [15]. EEG signal is non-stationary; thus, it is important to obtain a reliable estimate of the fractal dimension that selects invariant stationary signal segments [16] [17]. A common assumption in EEG processing methods that require stationarity is the presence of stationarity in short segments of the time series [18]. Hazarika's work [19] found that a one-second segment of the fixed length had approximately stationary characteristics. Therefore, to estimate the fractal dimension of the fixed length 1-second segments (256 samples) were used. The fractal dimension was estimated for all channels and then averaged for the entire EEG recording.

\subsection{Permutation Entropy}

In information theory and communication, entropy is considered the amount of uncertainty within a random variable. If the entropy is 0 , then the system is predictable. We used a simple complexity measure, which is easily calculated for any type of time series, be it regular, chaotic or noisy. We considered all $n$ ! permutations $\pi$ of order $n$, which are considered here as possible order types of $n$ different numbers. For each $\pi$ in $S n$, we determined the relative frequency $p(p i)$. The permutation entropy of the order $n \geq 2$ is defined as

$$
H(n)=-\sum p(\pi) \log p(\pi)
$$

where the sum contains all $n$ ! permutations $\pi$ of the order $n$ [20]. The permutation entropy was estimated as a non-segmented EEG signal in each channel and then averaged for all channels.

\subsection{Statistics}

The Wilcoxon signed-rank test was computed for both measures, fractal dimension and permutation entropy, before and during both types of meditation. The Bonferroni correction was used for multiple pairwise comparisons.

\section{Results}

For both types of meditation, global permutation entropy decreased (Table 1). In contrast, the fractal dimension for both types of meditation increased. This increase was statistically significant only in calming meditation (Table 2).

\section{Discussion}

Permutation entropy showed promise in differentiating between EEG recordings before and during both types of meditation (Table 1). Reduced permutation entropy during meditation indicates that the meditative state requires control to maintain the resource allocation of attention associated with inhibition of inappropriate stimuli. The reduction of permutation entropy may be due to more strongly linked oscillators or inactivation of previously active neural networks [21]. It is likely that this effect is influenced by the activities of the cingulate, which is

\begin{tabular}{|c|c|c|c|c|c|}
\hline \multirow[t]{2}{*}{ Type of Meditation } & \multirow{2}{*}{$\begin{array}{c}\text { Before Meditation } \\
\text { Mean }\end{array}$} & \multirow{2}{*}{$\begin{array}{c}\text { During Meditation } \\
\text { SD }\end{array}$} & \multicolumn{3}{|c|}{ p-value } \\
\hline & & & Mean & $S D$ & \\
\hline Calming meditation & 0.811 & 0.050 & 0.802 & 0.053 & $2.560 \times 10^{-4}$ \\
\hline Insight meditation & 0.817 & 0.048 & 0.797 & 0.052 & $2.888 \times 10^{-15}$ \\
\hline
\end{tabular}
included in the meditation process [22]. Can you imagine that a meditative experience accompanied by a reduc-

Table 1. Comparison of permutation entropy before and during both types of meditation. 
Table 2. Comparison of the fractal dimension before and during both types of meditation.

\begin{tabular}{cccccc}
\hline Type of Meditation & Before Meditation & During Meditation & \multicolumn{3}{c}{ p-value } \\
\hline & Mean & SD & Mean & SD \\
\hline Calming meditation & 1.415 & 0.066 & 1.434 & 0.051 & $1.394 \times 10^{-4}$ \\
Insight meditation & 1.436 & 0.042 & 1.439 & 0.042 & 0.768 \\
\hline
\end{tabular}

tion in permutation entropy turns “off” unnecessary neural network activity to maintain focus on internalised attention. According to the Hebb’s rule, a population of neurons forms a functional unit to process information, and at the same time, the number of activated neuronal populations can be regarded as an indicator of the complexity of neuronal computing operations in the brain [21] [23], which is reflected in the value of the permutation entropy. Increasing the value of the fractal dimension cannot be explained by increasing the amplitude of the signal due to EEG synchronisation in meditation, because the Sevcik fractal dimension, unlike the Katz fractal dimension, is insensitive to the amplitude of the evaluated signal [7]. This result can be interpreted as an increase in self-similarity of EEG signals. This phenomenon may be related to increased self-organisation of the hierarchical structure oscillators in the brain during meditation.

Improved control of attentional control [24] was associated with a reduction in the complexity of the EEG, while various normal and pathological conditions associated with loss of attention [25] [26] and a divergent way of thinking [27] have been associated with increasing complexity mainly in the frontal areas. These findings are consistent with a significant increase in the fractal dimension of meditation of calm that requires focused attention, but during insight meditation, activating the network, most likely mirrorcells, produces significant changes from pre-meditation signals.

The weakness of the work performed is missing statistical testing compared to the surrogate data. This technique allows for a stationary signal to distinguish linearly filtered noise from non-linear time series. However, for non-stationary signals, this technique can only reflect the non-stationarity of the signal. This method could be used in testing the FD, which requires a stationary signal, and its estimates were used in the one-second segments that were considered as acceptably stationary [19]. The permutation entropy, however, does not require a stationary signal [8], and the non-stationary segments of the EEG signal that were estimated by this method might cause the failure of that approach.

\section{Conclusion}

As this study verified the existence of statistically significant differences between certain features of EEG time series before meditation and during both types of meditation, further study should attempt to verify this difference on out-of-sample data sets. Following verification on multiple data sets, machine learning algorithms, such as artificial neural networks, decision trees and other classifiers, can utilize these features as attributes to detect calming and insight meditation.

\section{References}

[1] Malinowski, P. (2013) Neural Mechanisms of Attentional Control in Mindfulness Meditation. Frontiers in Neuroscience, 7, 8. http://dx.doi.org/10.3389/fnins.2013.00008

[2] Baer, R.A. (2005) Mindfulness-Based Treatment Approaches: Clinician’s Guide to Evidence Base and Applications. Academic Press, New York.

[3] Didonna, F. (2009) Clinical Handbook of Mindfulness. Springer, New York.

[4] Bhattacharya, J. (2000) Complexity Analysis of Spontaneous EEG. Acta Neurobiologiae Experimentalis, 60, $495-501$.

[5] Rapp, P.E., Albano, A.M., Schmah, A.M. and Farwell, L.A. (1993) Filtered Noise Can Mimic Low-Dimensional Chaotic Attractors. Physical Review E, 47, 2289-2297. http://dx.doi.org/10.1103/PhysRevE.47.2289

[6] Klonowski, W., Jernajczyk, W., Niedzielska, K., Rydz, A. and Stepień, R. (1999) Quantitative Measure of Complexity of EEG Signal Dynamics. Acta Neurobiologiae Experimentalis, 59, 315-321.

[7] Raghavendra, B.S. and Narayana Dutt, D. (2010) Computing Fractal Dimension of Signals Using Multiresolution BoxCounting Method. World Academy of Science, Engineering and Technology, 61, 1223-1238.

[8] Kreuzer, M., Kochs, E.F., Schneider, G. and Jordan, D. (2014) Non-Stationarity of EEG during Wakefulness and Anaesthesia: Advantages of EEG Permutation Entropy Monitoring. Journal of Clinical Monitoring and Computing. 
http://dx.doi.org/10.1007/s10877-014-9553-y

[9] Theiler, J., Eubank, S., Longtin, A., Galdrikian, B. and Farmer, J.D. (1992) Testing for Nonlinearity in Time Series: The Method of Surrogate Data. Physica D, 58, 77-94. http://dx.doi.org/10.1016/0167-2789(92)90102-S

[10] Rombouts, S.A.R.B., Keunen, R.W.M. and Stam, C.J. (1995) Investigation of Nonlinear Structure in Multichannel EEG. Physics Letters A, 202, 352-358. http://dx.doi.org/10.1016/0375-9601(95)00335-Z

[11] Stam, C.J., Breakspear, M., van Cappellen van Walsum, A.M. and van Dijk, B.W. (2003) Nonlinear Synchronization in EEG and Whole-Head MEG Recordings of Healthy Subjects. Human Brain Mapping, 19, 63-78. http://dx.doi.org/10.1002/hbm.10106

[12] Schreiber, T. and Schmitz, A. (2000) Surrogate Time Series. Physica D, 142, 346-382. http://dx.doi.org/10.1016/S0167-2789(00)00043-9

[13] Dunn, B.R., Hartigan, J.A. and Mikulas, W.L. (1999) Concentration and Mindfulness Meditations: Unique Forms of Consciousness? Applied Psychophysiology and Biofeedback, 24, 147-165. http://dx.doi.org/10.1023/A:1023498629385

[14] Aftanas, L.I. and Golocheikine, S.A. (2002) Non-linear Dynamic Complexity of the Human EEG during Meditation. Neuroscience Letters, 330, 143-146. http://dx.doi.org/10.1016/S0304-3940(02)00745-0

[15] Sevcik, C. (2006) On Fractal Dimension of Waveforms. Chaos, Solitons \& Fractals, 28, 579-580. http://dx.doi.org/10.1016/j.chaos.2005.07.003

[16] Hara, S., Kawaharaa, Y., Washioa, T., von Bnau, P., Tokunagac, T. and Yumotot, K. (2012) Separation of Stationary and Non-stationary Sources with a Generalized Eigenvalue Problem. Neural Networks, 33, 7-20. http://dx.doi.org/10.1016/j.neunet.2012.04.001

[17] Von Bunau, P., Meinecke, F., Kiraly, F. and Robert-Muller, K. (2009) Finding Stationary Subspaces in Multivariate Time Series. Physical Review Letters, 103, Article ID: 214101. http://dx.doi.org/10.1103/physrevlett.103.214101

[18] Quiroga-Lombard, C., Hass, J. and Durstewitz, D. (2013) A Method for Stationarity-Segmentation of Spike Train Data with Application to the Pearson Cross-Correlation. Journal of Neurophysiology, 110, 562-572. http://dx.doi.org/10.1152/jn.00186.2013

[19] Hazarika, N., Tsoi, A.C. and Sergejew, A.A. (1997) Nonlinear Considerations in EEG Signal Classification. IEEE Transactions on Signal Processing, 45, 829-936. http://dx.doi.org/10.1016/j.neunet.2012.04.001

[20] Bandt, C. and Pompe, B. (2002) Permutation Entropy: A Natural Complexity Measure for Time Series. Physical Review Letters, 88, Article ID: 174102. http://dx.doi.org/10.1103/PhysRevLett.88.174102

[21] Lutzenberger, W., Preissl, H. and Pulvermuller, F. (1995) Fractal Dimension of Electroencephalographic Time Series and Underlying Brain Processes. Biological Cybernetics, 73, 477-482. http://dx.doi.org/10.1007/BF00201482

[22] Newberg, A., Alavi, A., Baime, M., Pourdehnad, M., Santanns, J. and Aquilli, E. (2001) The Measurement of Regional Cerebral Blood Flow during the Complex Cognitive Task of Meditation: A Preliminary SPECT Study. Psychiatry Research, 106, 113-122.

[23] Elbert, T., Ray, W.J., Kowalik, Z.J., Skinner, J.E., Graf, K.E. and Birbaumer, N. (1994) Chaos and Physiology: Deterministic Chaos in Excitable Cell Assemblies. Physiological Reviews, 74, 1-47.

[24] Molle, M., Marshall, L., Pietrowsky, R., Lutzenberger, W., Fehm, H.L. and Born, J. (1995) Dimensional Complexity of EEG Indicates a Right Fronto-Cortical Locus of Attentional Control. Journal of Psychophysiology, 9, 45-55.

[25] Lutzenberger, W., Elbert, T., Birbaumer, N., Ray, W.J. and Schupp, H. (1992) The Scalp Distribution of the Fractal Dimension of the EEG and Its Variation with Mental Tasks. Brain Topography, 5, 27-34. http://dx.doi.org/10.1007/BF01129967

[26] Schupp, H.T., Lutzenberger, W., Birbaumer, N., Miltner, W. and Braun, C. (1994) Neurophysiological Differences between Perception and Imagery. Cognitive Brain Research, 2, 77-86. http://dx.doi.org/10.1016/0926-6410(94)90004-3

[27] Molle, M., Marshall, L., Wolf, B., Fehm, H.L. and Born, J. (1999) EEG Complexity And Performance Measures of Creative Thinking. Psychophysiology, 36, 95-104. http://dx.doi.org/10.1017/S0048577299961619 


\section{Appendix}

\section{Instructions}

The participants (meditating or not) were provided with these instructions at least one week before the experiment to familiarise themselves with the experimental structure and the respective types of meditation. The participants in the control group will try both types of meditation at least twice before the experiment, i.e., 2 times of 30 minutes of calming meditation and 2 times of 30 minutes of insight meditation. All questions should be answered before the experiment. Unless it is indeed necessary, the participants should not talk during the experiment.

The experiment is not painful nor dangerous.

Prior to the experiment (preferably while at home), please wash your hair (as sebum increases resistance under the electrodes). Bring a towel with you so that you can wash your hair after the experiment because you will have gel residue that will have been used for the procedure.

If you are used to sitting on a meditation pillow while meditating, please bring it with you. A mat is available but not a pillow. It is, of course, possible to sit on a chair during the experiment.

\section{Adaptation}

Sit down in a relaxed position with a straight back. Put your hands on your knees or in your lap and relax your shoulders. If you are sitting on a chair, do not lean too much on the backrest and ensure your entire soles are touching the ground. Close your eyes, relax and get used to this position, the feeling of the device on your head, the surrounding sounds and the environment. The position should not be too tense, but you should not sit slouched either. If the position is uncomfortable (you should not move with every twitch or itch), realize the discomfort and intention to change position and then slowly change the position while paying attention to the sequence of movements accompanying the changes. It is also possible to change the position in this way during the meditation.

\section{Calming Meditation}

Remain in a seated position and keep your eyes closed.

Keep your attention on the current moment and focus your attention on raising and lowering the abdominal wall while breathing in and out. Do not make an excessive effort, and do not try to keep your attention under any circumstances. Do not manipulate your breathing, i.e., do not accelerate, slow down, deepen, etc. Additionally, the point is not to think about or contemplate the breathing, etc. Follow the naturally occurring bodily sensations around the abdominal wall (movement, tension, feelings, etc.). When the abdominal wall goes up, you observe that it goes up, when it goes down, you observe that it goes down.

Your attention may be distracted by sounds, visual images, pleasant and unpleasant sensations in other parts of your body, e.g., pain. Your attention may also be distracted by emotions and moods (e.g., restlessness, irritation, boredom, sleepiness, tiredness, expectations, anxiety, etc.). The most frequent distraction will most likely concern thinking, e.g., "This is boring. Will I be able to sit this long? What purpose does this exercise serve? What have I been doing today? What do I still have to do today?” If anything distracts your attention from raising and lowering your abdominal wall, realize that you have been distracted and gently, without any excessive effort, streamline your attention back to the raising and lowering of the abdominal wall. Repeat this process continuously. Patiently and gently streamline your attention back to the raising and lowering of the abdominal wall in the present moment.

Note for meditators: If you are used to it, you may follow the sensations related to breathing in other parts of your body, e.g., air flow on the nostrils. It is also possible to follow the breathing not only as a bodily process but also as the so-called nimita, i.e., the mental symbol of breath.

\section{Break}

Stop the meditation, and for the next five minutes, remain in a seated position with your eyes closed. Do not speak.

After five minutes, you can open your eyes and disconnect from the device if you want to and take a slow 
walk or go to the restroom, etc. Speak only if absolutely necessary, and do not use your phone, work on the computer, etc.

The ten-minute break is followed by three minutes during which you will retake the meditative position, close your eyes, become relaxed and get ready for meditation.

\section{Insight Meditation}

Stay in the seated position with your eyes closed.

Keep your attention on the present moment, and mindfully register anything you are currently experiencing. Everything that distracted your attention from raising and lowering the abdominal wall during the calming meditation now serves as the object of attention. It may be your breathing, bodily sensations and other sensory perceptions (sounds, visual images, smells, or tastes), pleasant and unpleasant feelings, emotions and moods (irritation, restlessness, boredom, sleepiness, tiredness, expectations, anxiety, etc.), judgements (good/bad, I want/do not want it), or thinking. In addition, you may register the overall quality of the experience, the feelings related to being in this place and time (in this bodily position, in this room and building). If thinking occurs, register the actual process of thinking and the feelings of thinking, and do not get involved with the contents of the thoughts. If your attention is spread out and it is too difficult to maintain your attention on the present moment, you may focus your attention on breathing in and out for a while, just as during calming meditation. Do not make an excessive effort or try to maintain your attention at any expense. Patiently and gently streamline your attention back to the present moment.

Note for meditators: If you are used to going back to the in-and-out breathing while practicing insight meditation, you may do so. However, pay careful attention to the nature of all stimuli that distract you and also to the tendency to return to breathing.

\section{Conclusion}

Stop the meditation, but for five minutes, remain in the seated position with your eyes closed. Do not speak. 
Scientific Research Publishing (SCIRP) is one of the largest Open Access journal publishers. It is currently publishing more than 200 open access, online, peer-reviewed journals covering a wide range of academic disciplines. SCIRP serves the worldwide academic communities and contributes to the progress and application of science with its publication.

Other selected journals from SCIRP are listed as below. Submit your manuscript to us via either submit@scirp.org or Online Submission Portal.
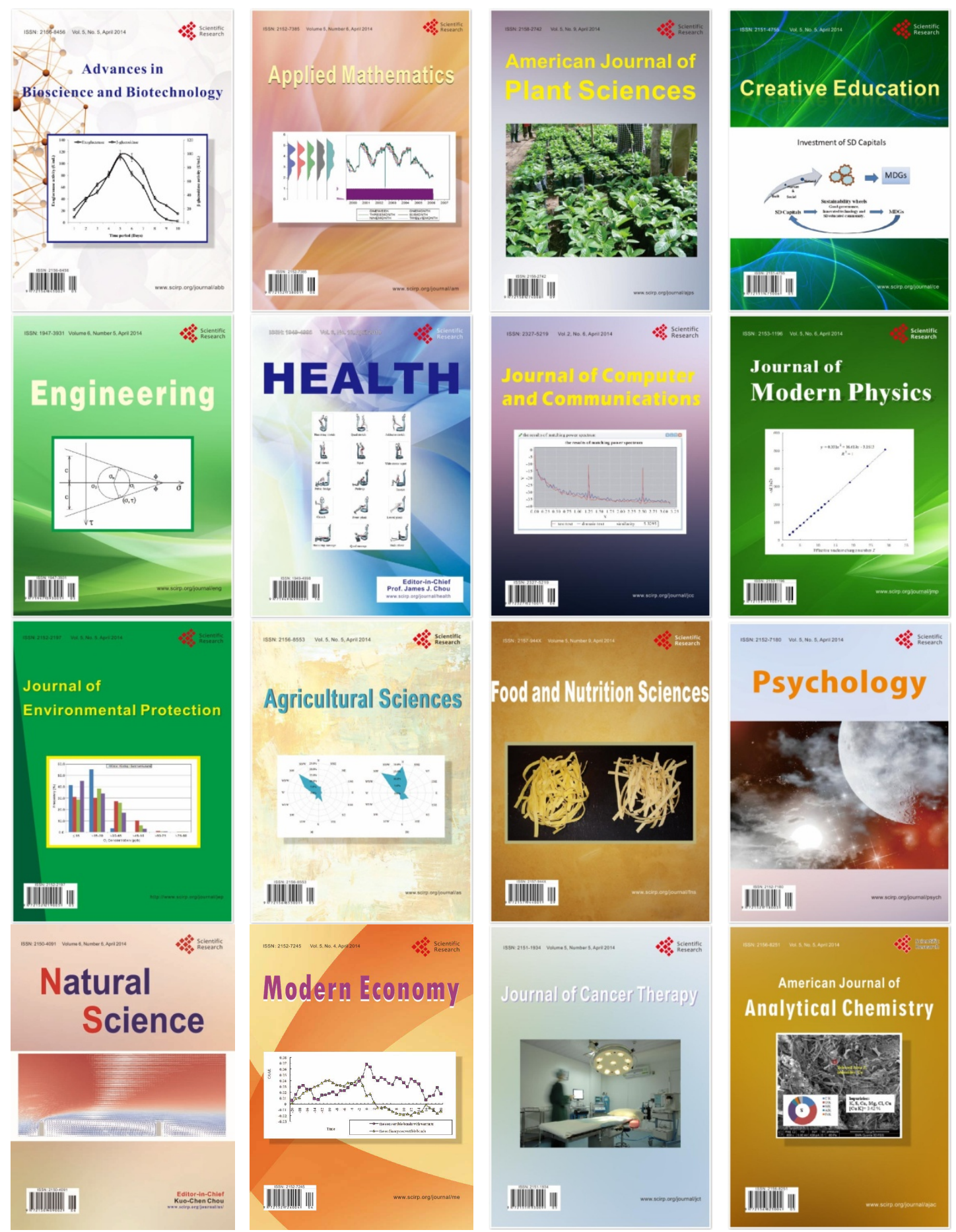